\title{
Morphometric study of lingual foramina in macerated mandibles to assist in implant placement in the anterior mandibular region
}

\author{
N.F. Deana ${ }^{1}$, P. Navarro², N. Alves ${ }^{3}$ \\ ${ }^{1}$ Master Programme in Dentistry, Faculty of Dentistry, Universidad de La Frontera, Temuco, Chile \\ ${ }^{2}$ Research Centre in Dental Sciences (CICO), Dental School, Universidad de La Frontera, Temuco, Chile \\ ${ }^{3}$ Research Centre in Applied Morphology (CIMA), Dental School, Universidad de La Frontera, Temuco, Chile
}

[Received: 13 March 2107; Accepted: 4 August 2017]

\begin{abstract}
The object of our study was to contribute to anatomical knowledge of this region with data on the prevalence, number and location of lingual foramina (LF) in dentate and edentate macerated mandibles from Brazilian individuals, differentiating them by sex and race. In this way we hope to help dental surgeons with their planning prior to implant placement in the anterior mandibular region. 103 macerated mandibles were analysed. The prevalence, number and location of LF were analysed in the median (MLF) and lateral (LLF) regions and the median (AMLF) and lateral (ALLF) alveolar process regions. Measurements for their location were taken with a digital calliper. $99 \%$ of the mandibles presented at least $1 \mathrm{MLF}$, $82.5 \%$ at least 1 LLF, and the frequency of ALLF was $67 \%$. In dentate mandibles, $M L F$ were located in the region superior to the genial spine, and in edentate mandibles in the regions superior and inferior to the genial spine. LLF were located in the middle region in both dentate and edentate mandibles. The height of the symphysis was significantly greater in dentate than in edentate cases. The distance from the alveolar crest (AC) to the MLF was significantly greater in dentate than in edentate mandibles. LF are constant structures, with MLF found more frequently than LLF. Mandibles which present a smaller measured distance from the base of mandible to AC present MFL and LLF closer to AC, implying a greater risk of complication during implant placement. (Folia Morphol 2018; 77, 2: 310-322)
\end{abstract}

Key words: lingual foramina, mandible, implant placement, morphometry

\section{INTRODUCTION}

In descriptions of surgical procedures involving the anterior mandibular region, the presence of lingual foramina and neurovascular bundles is frequently ignored or considered to be an anatomical detail associated with an unimportant clinical and surgical risk [23]. The incisive neurovascular bundle, which comes from the inferior alveolar neurovascular bundle, is responsible for the innervation and irrigation of the lower anterior teeth and the alveolar bone in the region. The incisive artery and the mental artery together irrigate the anterior inferior vestibular gum; while the sublingual artery (which anastomoses with the submental artery) and the mylohyoideus artery together irrigate the inferior lingual gum and the floor of the mouth [1]. The blood vessels that vascularise the floor of the mouth arrive very close to the lingual cortical on the median plane, where the lingual foramina

Address for correspondence: N. Alves, MD, PhD, Research Centre in Applied Morphology (CIMA), Dental School, Universidad de La Frontera, 1145 Francisco Salazar Avenue, PO-BOX 54-D, Temuco, Chile, e-mail: nilton.alves@ufrontera.cl 
can be found [2, 23]. Lustig et al. [13], in a study done with Doppler ultrasound and periapical X-rays, identified and carried out a quantitative evaluation of the arterial supply to the anterior mandibular region. These authors state that apart from the incisive, sublingual, facial and mental arteries and their muscle branches, there is an artery to the lingual foramen, located between the two superior genial spines. They suggest that this artery originates in the anastomosis of the two sublingual arteries, forming a single short vessel which is the principal source of irrigation for the anterior mandibular region. The authors even suggest that Doppler ultrasound examination should be included in pre-operatory procedures for genioplasty, to assess the arterial supply to the mental region. The principal complications in inserting implants in the mandible are paraesthesia and haemorrhage caused by injury to the inferior alveolar neurovascular bundle [9]. Blood vessels in the anterior mandibular lingual region are normally of 1-2 mm diameter; vessels of this calibre would allow up to $400 \mathrm{~mL}$ of intravascular blood to be drained in $30 \mathrm{~min}$ [2]. Woo et al. [24] reported a case of a haemorrhage after implant placement in the region of the lower central incisor when the lingual cortex was perforated, injuring the sublingual artery. According to Hofschneider et al. [4], the sublingual artery can be observed in the anterior mandibular region in up to $70 \%$ of individuals, while in $41 \%$ of individuals there is a branch of the submental artery perforating the mylohyoideus muscle in the same region. Extensive arterial anastomoses have been reported in the anterior mandibular lingual region. These arteries may lie superficially in atrophic cases and can be an important source of haemorrhage if traumatised during implant placement. Haemorrhages in the anterior mandibular region (immediate or delayed) caused by implant placement may lead to serious respiratory complications even including death of the patient [11].

Anatomical knowledge is essential for safe surgical procedures, avoiding the risk of complications in implant placement in the anterior mandibular region. The object of our study was to contribute to anatomical knowledge of this region with data on the prevalence, number and location of lingual foramina in dentate and edentate macerated mandibles from Brazilian individuals, differentiating them by sex and race. In this way we hope to help dental surgeons with their planning prior to implant placement in the anterior mandibular region.

\section{MATERIALS AND METHODS}

\section{Sample}

One hundred and three macerated mandibles were used in the present study, taken from Brazilian adult individuals of both sexes, black and white skin colours, aged between 20 and 89 years. They belonged to the Department of Morphology and Genetics, UNIFESP (Brazil). All the mandibles used in this study were identified by country of origin, sex and skin colour. There were 39 females (mean age: 39.43 years), and 64 males (mean age: 44.77 years). Of the total sample of 103 mandibles, 55 were from black individuals (mean age: $\mathbf{3 7 . 3 6}$ years), being 28 males and 27 females, and 48 from white individuals (mean age: 47.21 years), being 12 females and 36 males. Mandibles for which there was no information on sex and race, damaged mandibles and those which presented any kind of pathology were excluded from this study. The mandibles included in the study were divided between dentate and completely edentate samples.

\section{Analysis of the frequency, location and distance measurements of MLF and LLF}

After the foramina had been identified visually, a flexible wire was used to confirm their presence. Foramina located close to the median line (area of mandibular symphysis) superior, middle and inferior to the genial spine (GS), were called median lingual foramina (MLF); foramina located laterally, between the central incisor and the canine, were called lateral lingual foramina (LLF) [15]. Foramina located in the region of the alveolar process were analysed separately and called alveolar MLF (AMLF) when located between the central incisors, and alveolar LLF (ALLF) when located between the central incisor and the canine (Fig. 1). We analysed the frequency and number of foramina located in the median, lateral and alveolar (median and lateral) regions. The following distances were measured with a digital calliper, according to Natsis et al. [19]:

1. AC-BM - alveolar crest to base of mandible.

2. $A C-M_{\mathrm{s}}$ - alveolar crest to median lingual foramina (superior).

3. $\mathrm{AC}^{-\mathrm{MLF}_{\mathrm{m}}}$ - alveolar crest to median lingual foramina (middle).

4. AC-MLF $\mathrm{F}_{\mathrm{i}}$ - alveolar crest to median lingual foramina (inferior).

5. $\mathrm{BM}-\mathrm{MLF}_{\mathrm{s}}$ - base of mandible to median lingual foramina (superior). 


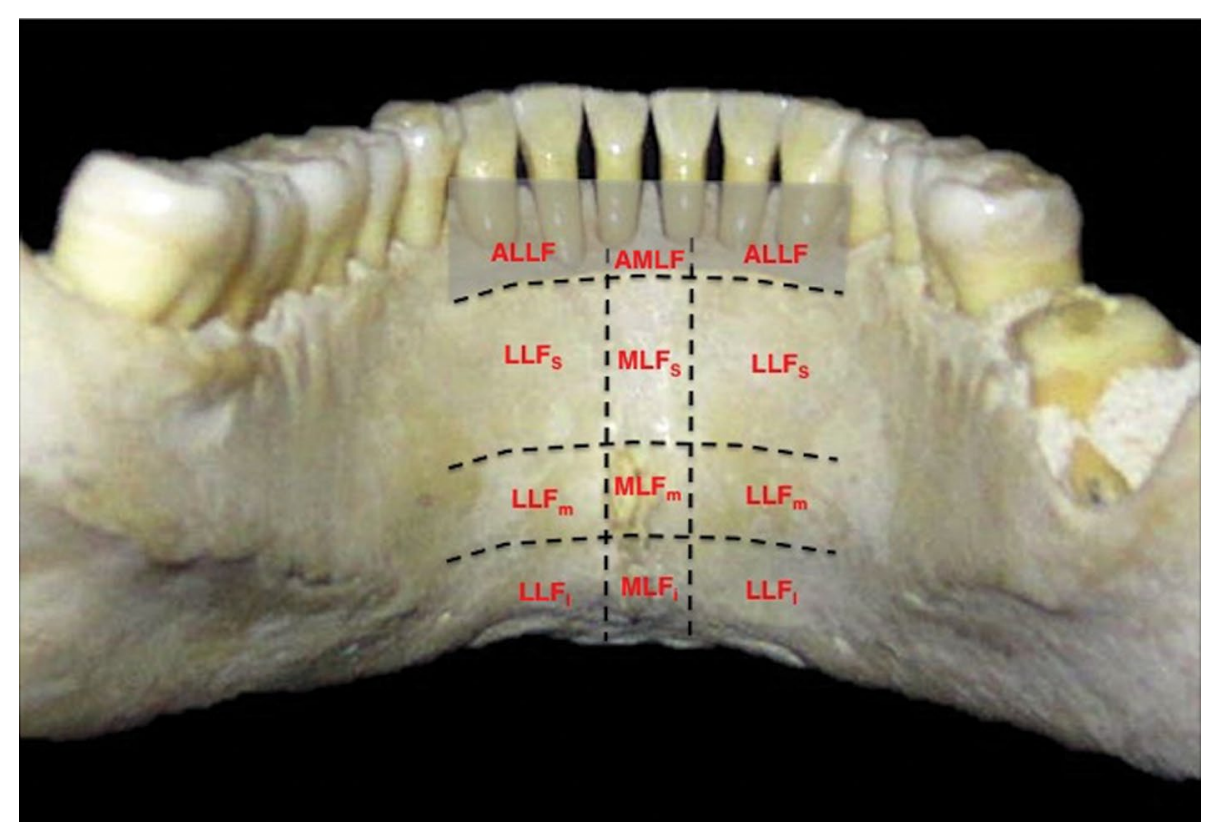

Figure 1. Image of the lingual face of the anterior mandibular region showing possible locations of lingual foramina; MLF — foramina located close to the median line (area of the mandibular symphysis) superior, middle and inferior to genial spine (GS); LLF — foramina located laterally of the GS (superior, middle and inferior) between the central incisor and the canine (superior, middle and inferior); AMLF — foramina located in the region of the alveolar process between the two central incisors; ALLF — foramina located in the region of the alveolar process between the central incisor and canine.

6. BM-MLF $m$ - base of mandible to median lingual foramina (middle).

7. $B M-M L F_{i}$ - base of mandible to median lingual foramina (inferior).

8. AC-LLF - alveolar crest to lateral lingual foramina, right $\left({ }_{R}\right)$ and left $\left({ }_{L}\right)$ sides.

9. BM-LLF - base of mandible to lateral lingual foramina, right $\left({ }_{R}\right)$ and left $\left({ }_{L}\right)$ sides.

10. GS-LLF - genial spine to lateral lingual foramina, right $\left({ }_{R}\right)$ and left $\left({ }_{L}\right)$ sides.

11. ALLF-AC - alveolar lateral lingual foramina to alveolar crest, right $\left({ }_{R}\right)$ and left $\left(_{L}\right)$ sides.

12. ALLF-BM - alveolar lateral lingual foramina to base of mandible, right $\left({ }_{R}\right)$ and left $\left(_{L}\right)$ sides.

The distances measured and the frequency of lingual foramina were analysed by sex and race.

\section{Statistical analysis}

The data collected were recorded in a Microsoft Office Excel spread-sheet and analysed using SPSS Statistics for Windows (version 20.0, IBM Corp., Armonk, NY). The data were subjected to descriptive analysis to determine the means and standard deviations, applying the Kolgomorov-Smirnov normality test, Pearson's $\chi^{2}$ test, t-test for independent samples, Mann-Whitney $U$ test for independent samples and Spearman's correlation coefficient for correlations between the measurements found for the distances analysed. A value of $p<0.05$ was selected as the threshold of statistical significance.

\section{RESULTS}

\section{Analysis of the prevalence and number of lingual foramina}

Of all the mandibles analysed in this study, 99\% presented at least 1 MLF. We observed that all the edentate mandibles presented at least $1 \mathrm{MLF}$ while $1.2 \%$ of the dentate mandibles did not present at least 1 MLF. In general, dentate mandibles presented 1 or $2 \mathrm{MLF}$, while the great majority of edentate mandibles presented 2 MLF (Figs. 2, 3).

We found that $82.5 \%$ of mandibles presented at least 1 LLF on the right and/or left side. In dentate mandibles the number of absences was greater on the right side $(45.9 \%)$, while in edentate mandibles the number of absences was greater on the left side (55.6\%). Up to 3 LLF were found in each hemimandible of edentate individuals and up to 2 LLF in hemimandibles of dentate individuals; the most common pattern was 1 LLF in each hemimandible, in both dentate and edentate individuals (Figs. 2, 4).

Median lingual foramina were more frequent than LLF in both dentate and edentate individuals; 


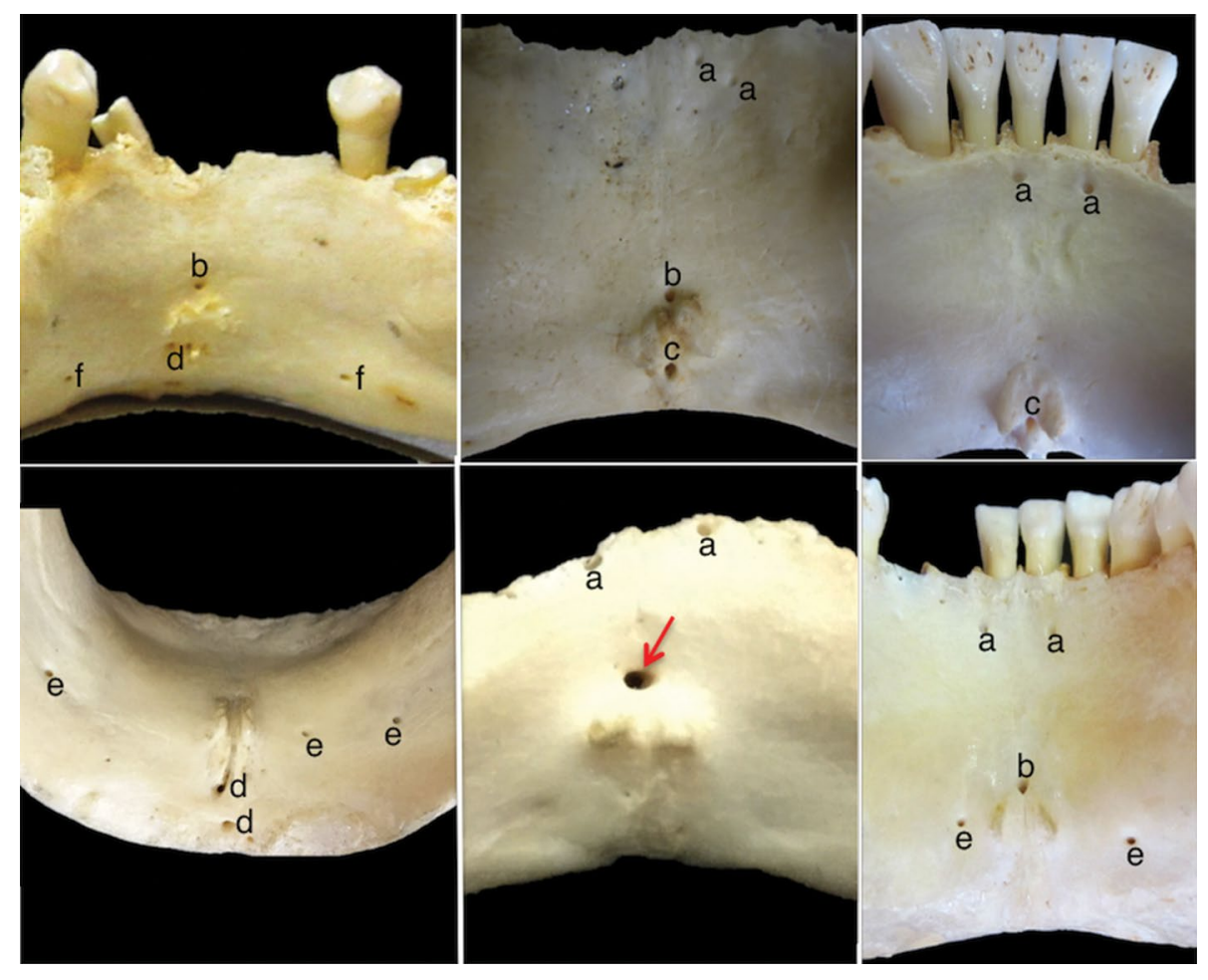

Figure 2. Image illustrating lingual foramina: $a$ - alveolar lateral lingual foramina (ALLF); $b$ - median lingual foramina superior to the genial spine (GS) $\left(\mathrm{MLF}_{\mathrm{s}}\right) ; \mathrm{c}$ - median lingual foramina middle to $\mathrm{GS}\left(\mathrm{MLF}_{\mathrm{m}}\right) ; \mathrm{d}$ - median lingual foramina inferior to $\mathrm{GS}\left(\mathrm{MLF}_{\mathrm{i}}\right)$; $\mathrm{e}$ - lateral lingual foramina middle to $G S\left(\operatorname{LLF}_{\mathrm{m}}\right) ; \mathrm{f}$ — lateral lingual foramina inferior to $\mathrm{GS}\left(\mathrm{LLF}_{\mathrm{i}}\right)$; arrow — large calibre $\mathrm{MLF}_{\mathrm{s}}$.

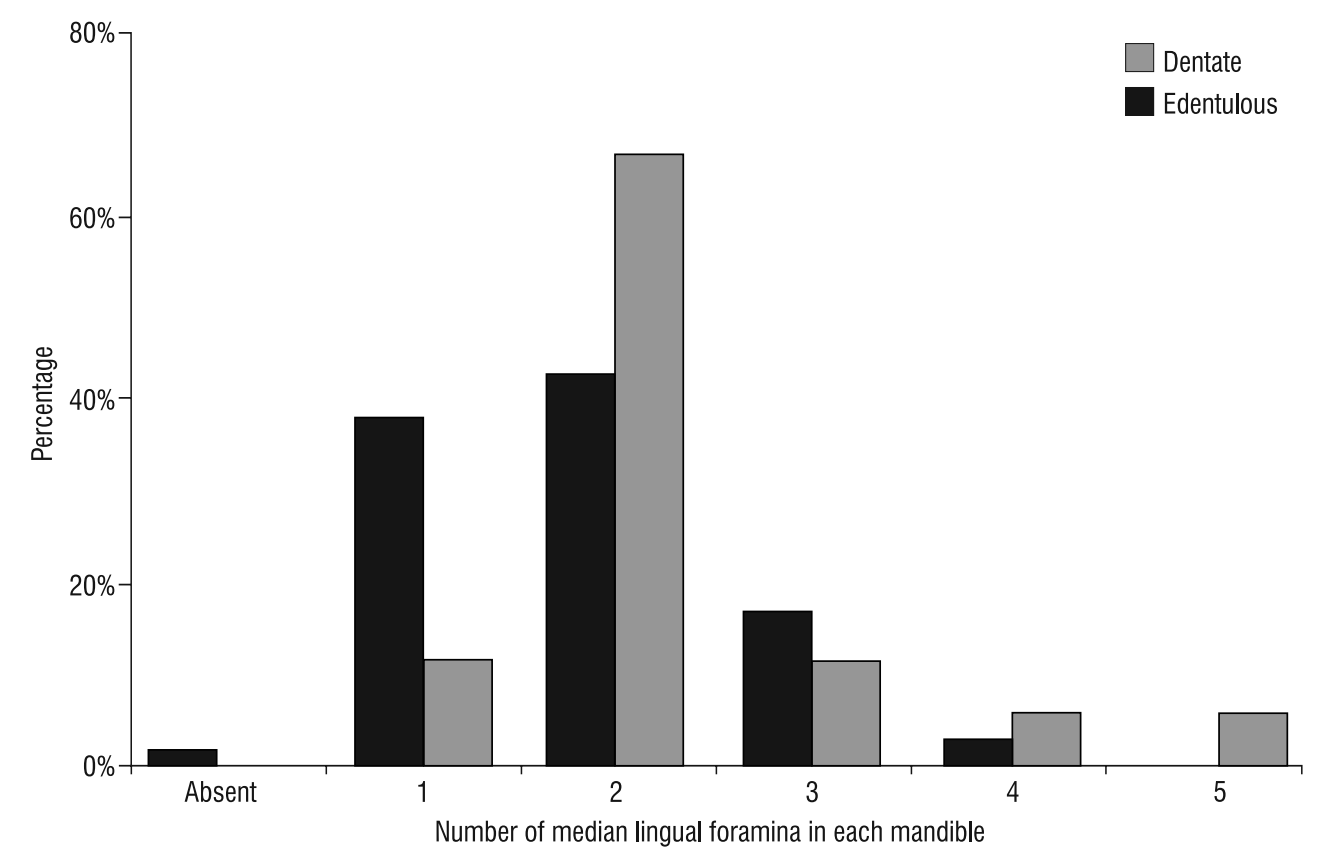

Figure 3. Frequency (\%) and number of median lingual foramina found in dentate and edentate mandibles.

however, no statistically significant difference was observed. Furthermore, no statistically significant difference was observed in the analyses by sex and race for the prevalence of MLF and LLF. 


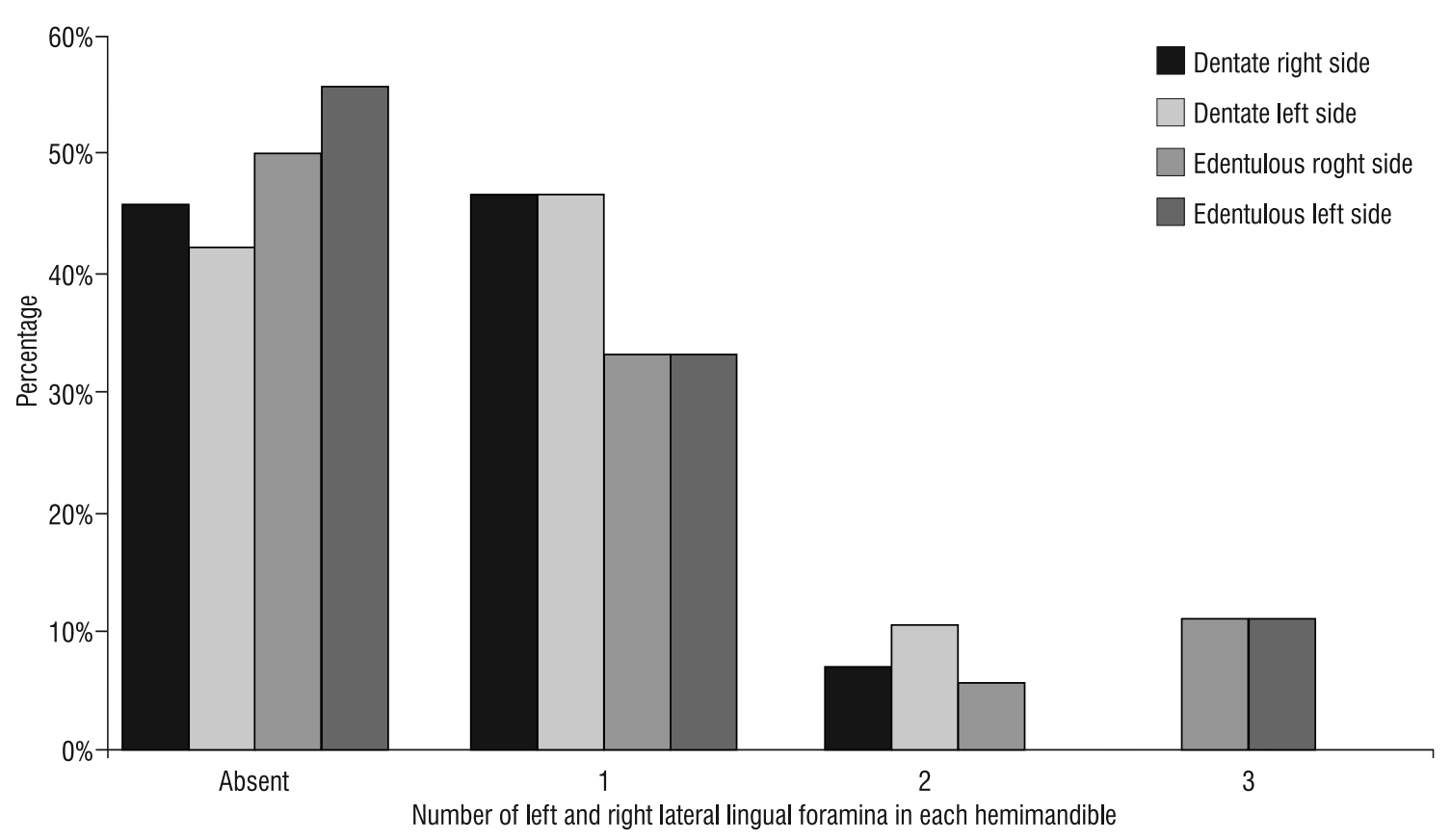

Figure 4. Frequency (\%) and number of left and right lateral lingual foramina in dentate and edentate mandibles.

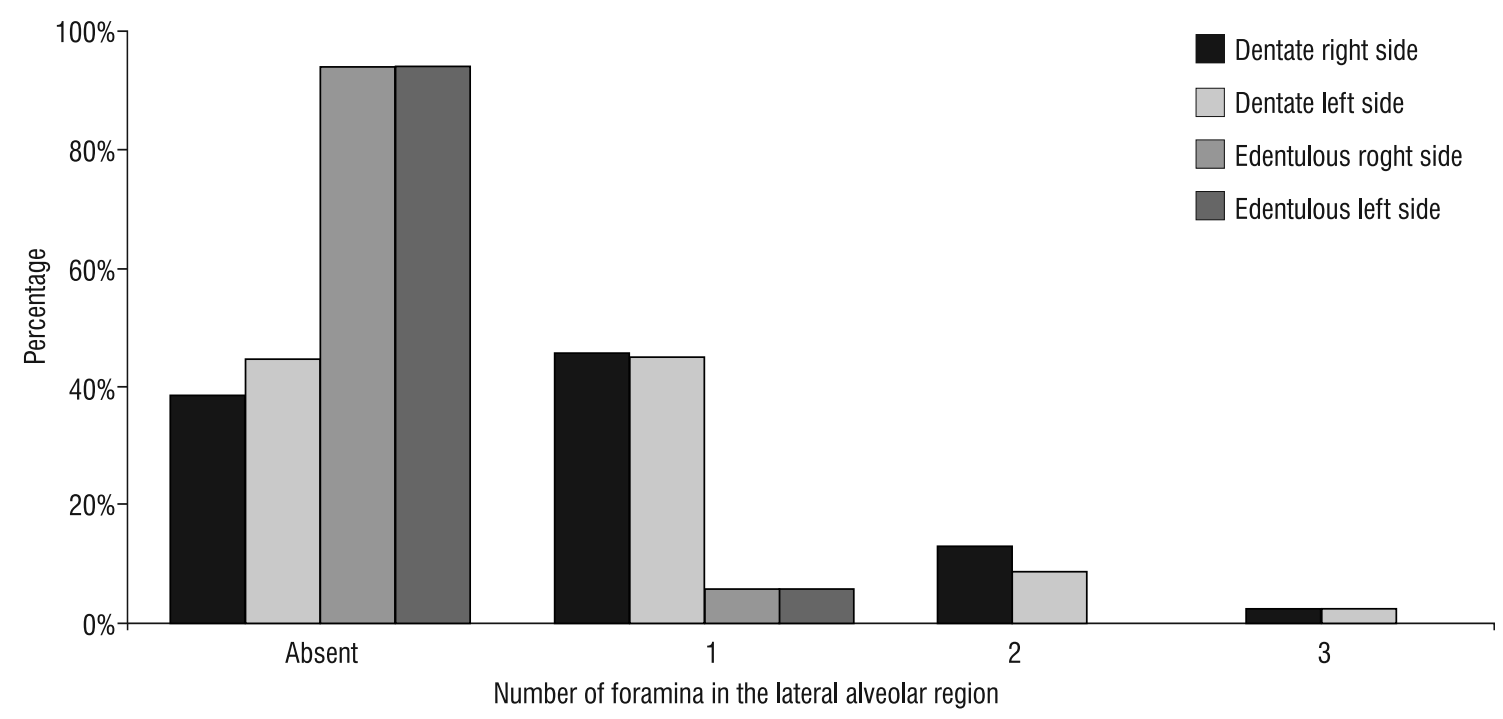

Figure 5. Frequency (\%) and number of foramina in the lateral alveolar region of dentate and edentate mandibles.

The frequency of ALLF was $67 \%$. In dentate mandibles the number of absences was greater on the left $(44.7 \%)$ than the right side (38.8\%); in edentate mandibles, ALLF were present in only 1 mandible, in which they presented bilaterally. In dentate individuals, up to 3 ALLF were found in each hemimandible; however, the presence of a single ALLF was significantly more frequent (Figs. 2, 5). No mandible was found with AMLF between the central incisors. ALLF were more frequent on the right side than on the left side in black individuals ( $p=0.009)$, and more prevalent in men than in women $(p=0.01)$.

\section{Analysis of the locations of MLF and LLF}

In dentate individuals, MLF were very frequently located in the region superior to the GS in all groups studied. In edentate males and white individuals, middle MFL were significantly less frequent than MFL, and $\mathrm{MFL}_{\mathrm{i}}$ (Table 1). Superior MFL were more frequent in dentate black males, black females, white males and 
Table 1. Location of median lingual foramina in the anterior mandibular region, separated by sex and skin colour

\begin{tabular}{|c|c|c|c|c|c|c|c|c|}
\hline & \multicolumn{4}{|c|}{ Dentate mandibles ( $n / \%$ ) } & \multicolumn{4}{|c|}{ Completely edentate mandibles (n/\%) } \\
\hline & Superior & Middle & Inferior & Total & Superior & Middle & Inferior & Total \\
\hline Black individuals & $46 / 54.8$ & $13 / 15.5$ & $25 / 29.7$ & $84 / 100^{* * *}$ & $7 / 35$ & $4 / 20$ & $9 / 45$ & $20 / 100$ \\
\hline White individuals & $34 / 48.6$ & $10 / 14.2$ & $26 / 37.2$ & $70 / 100^{* * *}$ & $10 / 47.6$ & $2 / 9.5$ & $9 / 42.8$ & $21 / 100^{*}$ \\
\hline Males & $50 / 50.5$ & $18 / 18.2$ & $31 / 31.3$ & $99 / 100^{* * *}$ & $9 / 50$ & 2/61.1 & $7 / 38.9$ & $18 / 100^{* *}$ \\
\hline Females & $30 / 54.5$ & $5 / 9.1$ & $20 / 36.4$ & $55 / 100 * * *$ & $8 / 34.9$ & $4 / 17.4$ & $11 / 47.8$ & $23 / 100$ \\
\hline White males & $26 / 47.3$ & $10 / 18.2$ & $19 / 34.5$ & $55 / 100$ & $8 / 53.3$ & $2 / 13.3$ & $5 / 33,4$ & $15 / 100$ \\
\hline Black males & $23 / 54.5$ & $8 / 18.5$ & $12 / 27.9$ & $43 / 100$ & $1 / 50.0$ & $0 / 0.0$ & $1 / 50.0$ & $2 / 100$ \\
\hline White females & $10 / 55.6$ & $1 / 5.6$ & $7 / 38.8$ & $18 / 100$ & $2 / 40.0$ & $0 / 0.0$ & $3 / 60.0$ & $5 / 100$ \\
\hline Black females & $21 / 55.3$ & $4 / 10.5$ & $13 / 34.2$ & $38 / 100$ & $6 / 31.6$ & $4 / 21.0$ & $9 / 47.4$ & $19 / 100$ \\
\hline
\end{tabular}

${ }^{*} p=0.01 ;{ }^{* *} p=0.03 ;{ }^{* * *} p<0.0001$

Table 2. Location of lateral lingual foramina in the anterior mandibular region

\begin{tabular}{|c|c|c|c|c|c|c|c|c|}
\hline & \multicolumn{4}{|c|}{ Dentate mandibles $(\mathrm{n} / \%)$} & \multicolumn{4}{|c|}{ Completely edentate mandibles (n/\%) } \\
\hline & Superior & Middle & Inferior & Total & Superior & Middle & Inferior & Total \\
\hline Black individuals & $3 / 4.3$ & $59 / 84.3$ & $8 / 11.4$ & $70 / 100^{* *}$ & 2/10.5 & $17 / 89.5$ & $0 / 0.0$ & $19 / 100^{* *}$ \\
\hline White individuals & $0 / 0.0$ & $31 / 77.5$ & 9/22.5 & $40 / 100^{* *}$ & $2 / 25$ & $5 / 62.5$ & $1 / 12.5$ & $8 / 100^{*}$ \\
\hline Males & $2 / 2.7$ & $59 / 80.8$ & $12 / 16.5$ & $73 / 100^{* *}$ & $2 / 25$ & $6 / 75$ & $0 / 0.0$ & $8 / 100^{*}$ \\
\hline Females & $1 / 2.7$ & $31 / 83.8$ & $5 / 13.5$ & $37 / 100^{* *}$ & $2 / 10.5$ & $16 / 84.2$ & $1 / 5.3$ & $19 / 100^{* *}$ \\
\hline White males & $0 / 0.0$ & $20 / 69.0$ & $9 / 31.0$ & $29 / 100$ & 2/28.6 & $5 / 71.4$ & $0 / 0.0$ & $7 / 100$ \\
\hline Black males & $0 / 0.0$ & $36 / 94.7$ & $2 / 5.3$ & $38 / 100$ & $0 / 0.0$ & $1 / 100$ & $0 / 0.0$ & $1 / 100$ \\
\hline White females & $6 / 35.3$ & $11 / 64.7$ & $0 / 0.0$ & $17 / 100$ & $0 / 0.0$ & $0 / 0.0$ & $1 / 100$ & $1 / 100$ \\
\hline Black females & $1 / 3.8$ & $20 / 76.9$ & $5 / 19.3$ & $26 / 100$ & $2 / 11.2$ & $16 / 88.8$ & $0 / 0.0$ & $18 / 100$ \\
\hline
\end{tabular}

${ }^{*} p \leq 0.01 ;{ }^{* *} p<0.0001$

white females; since in completely edentate individuals the commonest form was $\mathrm{MFL}_{\mathrm{s}}$ for white males, $\mathrm{MFL}_{\mathrm{i}}$ for black females and white females, while black males presented similar percentages for $\mathrm{MFL}_{\mathrm{s}}$ and $\mathrm{MFL}_{\mathrm{i}}$.

In both dentate and edentate individuals LLF $_{m}$ were significantly more frequent, while the presence of LLF in the region superior or inferior to the GS was very infrequent (Table 2). No statistical difference was found between races or sexes for the location of MLF and LLF. Dentate individuals and completely edentate black males, black females, white males and white females presented $\mathrm{LLF}_{\mathrm{m}}$ more frequently than $\mathrm{LLF}_{\mathrm{s}}$ and $\mathrm{LLF}_{\mathrm{i}}$ (Table 2).

\section{Analysis of the distance measurements found for MLF and LLF}

The mean values and standard deviation found for males, females, and black and white individuals are presented in Table 3. The mean values and standard deviation found for white males, white females, black males and black females are presented in Table 4.
The height of the symphysis, i.e. the dimension from the alveolar crest to the base of mandible (AC-BM), was significantly greater in dentate males, females and white individuals than in completely edentate males, females and white individuals ( $p=0.000$, $p=0.017$ and $p=0.000$, respectively). In dentate males the mean value for the $A C-M L F_{s}$ distance was around $10 \mathrm{~mm}$ greater than in completely edentate males $(p=0.000)$, and $6.82 \mathrm{~mm}$ greater in dentate than edentate white individuals $(p=0.000)$. Dentate white males presented mean values around $6.58 \mathrm{~mm}$ greater than completely edentate white males $(p=0.000)$.

The AC-MLF $F_{i}$ and $A C-L_{L} F_{R}$ distances were also significantly greater in dentate than edentate males ( $p=0.005$ and $p=0.004$, respectively), and in dentate than completely edentate white individuals ( $p=0.012$ and $p=0.004$, respectively). It was also observed that completely edentate white males presented significantly lower mean values than dentate 
Table 3. Mean values in millimetres and standard deviation (SD) found for dentate and completely edentate mandibles of males, females, black individuals and white individuals

\begin{tabular}{|c|c|c|c|c|c|c|c|c|c|c|c|c|c|c|c|c|}
\hline \multirow[t]{3}{*}{ Distances } & \multicolumn{4}{|c|}{ Males } & \multicolumn{4}{|c|}{ Females } & \multicolumn{4}{|c|}{ Black individuals } & \multicolumn{4}{|c|}{ White individuals } \\
\hline & \multicolumn{2}{|c|}{ Dentate } & \multicolumn{2}{|c|}{ Edentate } & \multicolumn{2}{|c|}{ Dentate } & \multicolumn{2}{|c|}{ Edentate } & \multicolumn{2}{|c|}{ Dentate } & \multicolumn{2}{|c|}{ Edentate } & \multicolumn{2}{|c|}{ Dentate } & \multicolumn{2}{|c|}{ Edentate } \\
\hline & Average & SD & Average & SD & Average & SD & Average & SD & Average & SD & Average & SD & Average & SD & Average & SD \\
\hline AC-BM & $32.25^{1}$ & 4.11 & $23.86^{1}$ & 3.82 & $31.01^{1}$ & 4.48 & $24.28^{1}$ & 6.74 & $33.36^{\dagger}$ & 4.33 & 27.22 & 6.78 & $30.14^{\dagger 1}$ & 3.46 & $23.29^{1}$ & 4.37 \\
\hline AC-MLF ${ }_{s}$ & $19.51^{1}$ & 4.43 & $9.90^{*}$ & 4.78 & 18.81 & 4.38 & $15.19^{*}$ & 4.87 & $20.96^{\dagger}$ & 4.28 & 16.16 & 6.33 & $17.36^{\dagger 1}$ & 3.72 & $10.54^{1}$ & 4.65 \\
\hline AC-MLF $_{\mathrm{m}}$ & 27.16 & 6.13 & 12.74 & 6.27 & 25.94 & 4.23 & - & - & 29.20 & 4.81 & - & - & 23.63 & 5.06 & 12.74 & 6.27 \\
\hline AC-MLF & $28.47^{1}$ & 3.96 & $21.44^{1}$ & 4.52 & 27.29 & 5.05 & 22.28 & 6.21 & 29.61 & 3.65 & 24.35 & 5.26 & $26.77^{1}$ & 4.47 & $20.92^{1}$ & 5.10 \\
\hline${\mathrm{BM}-M L F_{\mathrm{s}}}$ & 16.67 & 1.89 & 16.88 & 1.84 & 15.14 & 2.06 & 15.16 & 1.82 & 15.89 & 2.23 & 15.13 & 2.14 & 16.51 & 1.83 & 16.60 & 1.89 \\
\hline $\mathrm{BM}-\mathrm{MLF}_{\mathrm{m}}$ & 11.77 & 3.81 & 13.98 & 0.55 & 7.64 & 2.67 & - & - & 10.00 & 4.41 & - & - & 11.37 & 3.41 & 13.98 & 0.55 \\
\hline BM-MLF $_{\mathrm{i}}$ & 5.92 & 2.48 & 7.27 & 3.20 & 6.03 & 2.54 & 4.76 & 1.45 & 5.69 & 1.97 & 5.12 & 1.49 & 6.19 & 2.84 & 6.45 & 3.09 \\
\hline$A C-L_{R} F_{R}$ & $24.78^{1}$ & 4.39 & $13.75^{1}$ & 4.39 & 24.39 & 4.01 & 20.08 & 5.74 & 24.49 & 4.42 & 20.08 & 5.74 & $24.92^{1}$ & 4.02 & $13.75^{1}$ & 4.39 \\
\hline$A C-L L F_{L}$ & 25.13 & 4.32 & 17.33 & 8.45 & 23.05 & 4.37 & 17.12 & 8.06 & 24.37 & 3.96 & 22.82 & - & 24.73 & 5.05 & 16.34 & 7.94 \\
\hline BM-LLF $_{\mathrm{R}}$ & 8.97 & 3.39 & 12.03 & 4.96 & 8.70 & 1.62 & 9.94 & 1.95 & $9.75^{\dagger}$ & 3.08 & 9.94 & 1.95 & $7.51^{\dagger}$ & 2.07 & 12.03 & 4.96 \\
\hline BM-LLF & 9.72 & 3.22 & 11.05 & 3.14 & 8.13 & 2.78 & 6.46 & 2.66 & 9.30 & 42.99 & 8.34 & - & 9.19 & 3.46 & 9.98 & 3.86 \\
\hline $\mathrm{LLF}_{\mathrm{R}} \mathrm{-GS}$ & 13.77 & 3.47 & 10.86 & 7.30 & 12.01 & 5.44 & 12.90 & 5.31 & 13.11 & 34.47 & 12.90 & 5.31 & 13.58 & 3.52 & 10.86 & 7.30 \\
\hline $\operatorname{LLF}_{\mathrm{L}}-\mathrm{GS}$ & 11.93 & 4.40 & 10.17 & 8.28 & 12.80 & 5.46 & 9.05 & 5.14 & 13.43 & 4.62 & 12.69 & - & 10.63 & 4.45 & 9.37 & 7.65 \\
\hline Alveolar LLF-AC $C_{R}$ & 4.58 & 2.15 & - & - & 4.27 & 2.97 & 7.80 & - & 4.96 & 2.44 & 7.80 & - & 3.66 & 1.36 & - & - \\
\hline Alveolar LLF-AC & 4.95 & 2.59 & - & - & 4.46 & 2.69 & 5.64 & - & 5.47 & 2.75 & 5.64 & - & 3.48 & 1.66 & - & - \\
\hline Alveolar $\mathrm{LLF}_{\mathrm{R}}$-BM & 29.69 & 2.44 & - & - & 28.79 & 2.79 & 30.18 & - & 29.52 & 3.12 & 30.18 & - & 29.12 & 1.28 & - & - \\
\hline Alveolar LLF $F_{L}-B M$ & 29.73 & 2.23 & - & - & 28.96 & 2.86 & 29.95 & - & 29.30 & 2.82 & 29.95 & - & 29.77 & 1.58 & - & - \\
\hline
\end{tabular}

${ }^{*}$ Statistical significance between sexes; †Statistical significance between races; ${ }^{1}$ Statistical significance between dentate and edentate mandibles; AC — alveolar crest, BM — base of mandible; MLF — median lingual foramina (s - superior, $\mathrm{m}$ - middle, i — inferior); LLF — lateral lingual foramina ( $\mathrm{R}$ - right, $\mathrm{L}$ - left); GS — genial spine, (-) distance not calculate

Table 4. Mean values in millimetres and standard deviation (SD) found for dentate and completely edentate mandibles of males, females, black individuals and white individuals

\begin{tabular}{|c|c|c|c|c|c|c|c|c|c|c|c|c|c|c|c|c|}
\hline \multirow[t]{3}{*}{ Distances } & \multicolumn{4}{|c|}{ White males } & \multicolumn{4}{|c|}{ Black males } & \multicolumn{4}{|c|}{ White females } & \multicolumn{4}{|c|}{ Black females } \\
\hline & \multicolumn{2}{|c|}{ Dentate } & \multicolumn{2}{|c|}{ Edentate } & \multicolumn{2}{|c|}{ Dentate } & \multicolumn{2}{|c|}{ Edentate } & \multicolumn{2}{|c|}{ Dentate } & \multicolumn{2}{|c|}{ Edentate } & \multicolumn{2}{|c|}{ Dentate } & \multicolumn{2}{|c|}{ Edentate } \\
\hline & Average & SD & Average & SD & Average & SD & Average & SD & Average & SD & Average & SD & Average & SD & Average & SD \\
\hline AC-BM & $30.46^{1 \dagger}$ & 3.05 & 23.88 & 3.82 & $34.53^{\dagger}$ & 4.24 & - & - & 28.70 & 5.15 & 21.36 & 6.46 & 31.84 & 4.11 & 27.22 & 6.78 \\
\hline $\mathrm{AC}^{-M L F_{s}}$ & $17.551^{\dagger}$ & 3.25 & 9.90 & 4.78 & $21.95^{\dagger}$ & 4.56 & - & - & 16.58 & 5.70 & 13.73 & 2.79 & 19.66 & 3.66 & 16.15 & 6.33 \\
\hline$A C-M L F_{m}$ & 16.94 & 1.55 & 16.88 & 1.84 & 16.34 & 2.26 & - & - & 14.67 & 1.91 & 15.21 & 2.02 & 15.32 & 2.16 & 15.13 & 2.14 \\
\hline$A C-M L F_{i}$ & $23.63^{1 \dagger}$ & 5.06 & 12.74 & 6.27 & $32.45^{\dagger}$ & 2.79 & - & - & - & - & - & - & 25.94 & 4.23 & - & - \\
\hline BM-MLF $_{\mathrm{s}}$ & 11.37 & 3.41 & 13.98 & 0.55 & 12.37 & 4.84 & - & - & - & - & - & - & 7.64 & 2.67 & - & - \\
\hline BM- $\mathrm{MLF}_{\mathrm{m}}$ & 27.28 & 3.73 & 21.44 & 4.52 & 30.44 & 3.80 & - & - & 25.09 & 7.21 & 19.52 & 7.37 & 28.61 & 3.59 & 24.36 & 5.26 \\
\hline BM-MLF & 6.10 & 3.04 & 7.27 & 3.20 & 5.63 & 1.30 & - & - & 6.48 & 2.60 & 4.28 & 1.54 & 5.76 & 2.76 & 5.12 & 1.49 \\
\hline$A C-L L F_{R}$ & $25.23^{1}$ & 4.05 & 13.75 & 4.40 & 24.38 & 4.83 & - & - & 23.36 & 4.98 & - & - & 24.64 & 4.10 & 20.08 & 5.74 \\
\hline AC-LLF & 7.28 & 2.20 & 12.03 & 4.96 & 10.51 & 3.64 & - & - & 8.65 & 0.66 & - & - & 8.71 & 1.81 & 9.94 & 1.95 \\
\hline$B M-L_{R}$ & $24.59^{\dagger}$ & 4.92 & 17.33 & 8.45 & $25.67^{\dagger}$ & 3.77 & - & - & 25.58 & 7.96 & 11.42 & - & 22.43 & 3.63 & 22.82 & - \\
\hline BM-LLF $F_{L}$ & 9.00 & 3.60 & 11.05 & 3.14 & 10.44 & 2.77 & - & - & 10.30 & 3.30 & 4.58 & - & 7.59 & 2.60 & 8.34 & - \\
\hline $\mathrm{LLF}_{\mathrm{R}}-\mathrm{GS}$ & 14.44 & 2.73 & 10.86 & 7.30 & 13.21 & 4.01 & - & - & 9.27 & 4.98 & - & - & 12.93 & 5.70 & 12.9 & 5.31 \\
\hline $\mathrm{LLF}_{\mathrm{L}}-\mathrm{GS}$ & 11.58 & 3.95 & 10.17 & 8.28 & 12.35 & 5.09 & - & - & 4.93 & 3.12 & 5.41 & - & 14.77 & 3.85 & 12.69 & - \\
\hline Alveolar LLF-AC ${ }_{R}$ & $3.65^{\dagger}$ & 1.39 & - & - & $5.51^{\dagger}$ & 2.42 & - & - & 3.73 & 1.70 & - & - & 4.37 & 2.44 & 7.80 & - \\
\hline Alveolar LLF-AC $\mathrm{L}_{\mathrm{L}}$ & 29.13 & 1.36 & - & - & 30.25 & 3.15 & - & - & 29.05 & 0.95 & - & - & 28.74 & 3.04 & 30.18 & - \\
\hline Alveolar $\mathrm{LLF}_{\mathrm{R}}-\mathrm{BM}$ & $3.60^{\dagger}$ & 1.70 & - & - & $6.19^{\dagger}$ & 2.70 & - & - & 2.25 & - & - & - & 4.68 & 2.73 & 5.64 & - \\
\hline Alveolar $\mathrm{LLF}_{\mathrm{L}}-\mathrm{BM}$ & 25.93 & 1.46 & - & - & 29.91 & 2.82 & - & - & 32.05 & - & - & - & 28.65 & 2.81 & 29.95 & - \\
\hline
\end{tabular}

${ }^{*}$ Statistical significance between sexes; + Statistical significance between races; ${ }^{1}$ Statistical significance between dentate and edentate mandibles; AC — alveolar crest, BM — base of mandible; MLF — median lingual foramina ( - superior, $\mathrm{m}$ - middle, $\mathrm{i}$ - inferior); LLF — lateral lingual foramina ( $\mathrm{R}$ - right, $\mathrm{L}$ - left); $\mathrm{GS}$ - genial spine; (-) distance not calculate 
Table 5. Analysis of the correlation between the distance measurements analysed in the present study, by sex and race

\begin{tabular}{|c|c|c|c|c|c|c|c|c|}
\hline & \multicolumn{2}{|c|}{ Females } & \multicolumn{2}{|c|}{ Males } & \multicolumn{2}{|c|}{ Black individuals } & \multicolumn{2}{|c|}{ White individuals } \\
\hline & $\mathbf{r}$ & $\mathbf{p}$ & $\mathbf{r}$ & $\mathbf{p}$ & $\mathbf{r}$ & $\mathbf{p}$ & $\mathbf{r}$ & $\mathbf{p}$ \\
\hline $\mathrm{AC}-\mathrm{BM} \times \mathrm{AC}^{-M L F_{\mathrm{s}}}$ & 0.915 & 0.000 & 0.933 & 0.000 & 0.855 & 0.000 & 0.942 & 0.001 \\
\hline AC-BM $\times$ AC-MLF $_{m}$ & 0.900 & 0.037 & 0.940 & 0.000 & 0.933 & 0.000 & 0.767 & 0.016 \\
\hline AC-BM $\times$ AC-MLF & 0.952 & 0.000 & 0.964 & 0.000 & 0.930 & 0.000 & 0.950 & 0.000 \\
\hline$A C-B M \times A C-L L F_{R}$ & 0.762 & 0.002 & 0.637 & 0.000 & 0.517 & 0.014 & 0.895 & 0.000 \\
\hline $\mathrm{AC}-\mathrm{BM} \times \mathrm{AC}-\mathrm{LLF}_{\mathrm{L}}$ & 0.868 & 0.000 & 0.796 & 0.000 & 0.796 & 0.000 & 0.855 & 0.000 \\
\hline AC-MLF ${ }_{s} \times$ AC-MLF $_{m}$ & 0.900 & 0.037 & 0.916 & 0.000 & 0.917 & 0.001 & 0.762 & 0.028 \\
\hline 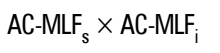 & 0.868 & 0.000 & 0.912 & 0.000 & 0.879 & 0.000 & 0.915 & 0.000 \\
\hline$A C-M L F_{s} \times A C-L L F_{R}$ & 0.734 & 0.007 & 0.706 & 0.000 & 0.482 & 0.027 & 0.949 & 0.000 \\
\hline$A C-M L F_{S} \times A C-L L F_{L}$ & 0.845 & 0.001 & 0.733 & 0.000 & 0.746 & 0.000 & 0.794 & 0.000 \\
\hline$A C-M L F_{m} \times A C-L^{2} F_{R}$ & 1.000 & $<0.05$ & 1.000 & $<0.05$ & 1.000 & $<0.05$ & -1.000 & NS \\
\hline$A C-M L F_{m} \times A C-L L F_{L}$ & 1.000 & $<0.05$ & 1.000 & $<0.05$ & 1.000 & $<0.05$ & 0.800 & NS \\
\hline $\mathrm{AC} \mathrm{MLF}_{\mathrm{i}} \times \mathrm{AC}^{-M L F_{\mathrm{m}}}$ & 1.000 & $<0.05$ & 1.000 & $<0.05$ & 1.000 & $<0.05$ & 1.000 & $<0.05$ \\
\hline$A C-M L F_{i} \times A C-L L F_{R}$ & 0.550 & NS & 0.744 & 0.001 & 0.448 & NS & 0.901 & 0.000 \\
\hline AC-MLF ${ }_{i} \times A C-L L F_{L}$ & 0.829 & NS & 0.771 & 0.001 & 0.771 & NS & 0.811 & 0.000 \\
\hline$A C-\mathrm{LLF}_{\mathrm{R}} \times \mathrm{AC}-\mathrm{LLF}_{\mathrm{L}}$ & 0.946 & 0.000 & 0.718 & 0.000 & 0.879 & 0.000 & 0.843 & 0.000 \\
\hline
\end{tabular}

r Spearman's rho; NS — no statistical significance; AC — alveolar crest; BM — base of mandible; MLF — median lingual foramina (s — superior, $\mathrm{m}$ — middle, i — inferior); LLF — lateral lingual foramina ( $\mathrm{R}$ - right, $\mathrm{L}$ - left)

white males, with a difference of $10.89 \mathrm{~mm}$ for AC$\mathrm{MLF}_{i}(p=0.019)$, and $11.48 \mathrm{~mm}$ for the $A C-$ LLF $_{R}$ distance $(p=0.005)$.

The $A C-M L F$ distance was significantly less in males than in females in completely edentate mandibles ( $p=0.04$ ); in completely edentate males the $\mathrm{MLF}_{\mathrm{s}}$ was located around $5.3 \mathrm{~mm}$ closer to the AC than in completely edentate females. No statistically significant difference was found between sides in either dentate or edentate individuals. Furthermore, in completely edentate white males the mean values were $7.65 \mathrm{~mm}$ smaller than in dentate white males $(p=0.001)$.

Differences between races were found in the AC-BM, AC-MLF $\mathrm{s}^{\prime}, \mathrm{BM}-\mathrm{LLF}_{\mathrm{R}}$ distances of dentate individuals; these distances were greater in black individuals than in white individuals $(p=0.007, p=0.006$ and $p=0.02$, respectively). It was also observed that the mean values found for black males were significantly greater than those found for white males for the AC-BM $(p=0.001), A C-M_{s}(p=0.001), A C-M L F_{i}$ $(p=0.019), B M-\operatorname{LLF}_{R}(p=0.009), \operatorname{ALLF}_{R}-A C(p=0.036)$, $\mathrm{ALLF}_{\mathrm{R}}-\mathrm{BM}(\mathrm{p}=0.019)$ distances.

\section{Analysis of correlation between distances}

Spearman's rho and the $p$ values for the correlations are presented in Tables 5 and 6 . The AC-MLF ${ }_{s^{\prime}}$
$A C-M L F_{m}, A C-M L F_{i}, A C-L L F_{R}$ and $A C-L_{L} F_{L}$ distances presented a strong or very strong positive correlation with the AC-BM distance in the whole sample, except for black individuals in whom the AC-LLF ${ }_{R}$ distance presented a moderate positive correlation with the $A C-B M$ distance. The $A C-M L F_{s^{\prime}} A C-M L F_{m^{\prime}} A C-M L F_{i}$, $A C-L F_{R^{\prime}} A C-L L F_{L}$ distances presented a strong or very strong positive correlation with the AC-BM distance for white males, black males and black females. The $A C-M^{\prime} F_{s^{\prime}} A C-M L F_{i}, A C-L_{L} F_{R}, A C-L_{L} F_{L}$ distances presented a strong or very strong positive correlation with the AC-BM distance for white females.

The $\mathrm{AC}-\mathrm{MLF}_{\mathrm{s}}$ distance presented a strong or very strong positive correlation with the $A C-M^{\prime} F_{m^{\prime}} A C-M L F_{i}$, $A C-L_{R}$ and $A C-$ LLF $_{L}$ distances, except in black individuals in whom a moderate positive correlation was observed between the AC-MLF $F_{s}$ and $A C-L_{R} F_{R}$ distances. The $A C-M L F_{S}$ distance presented a strong or very strong positive correlation with the AC-MLF ${ }_{m}, A C-M L F_{i}, A C-L_{R}$ and $A C-L L F_{L}$ distances for white males, black males and black females. The $A C-M_{S} F_{s}$ distance presented a strong or very strong positive correlation with the $A C-M L F_{i}, A C$ $L_{R} F_{R}$ and $A C-L L F_{L}$ distances for white females.

The AC-MLF ${ }_{m}$ distance presented a perfect positive correlation with the $A C-L_{L} F_{R}$ and $A C-L L F$ distances in all individuals, except for white individuals. There was a perfect positive correlation between $A C-M L F_{i}$ and 
Table 6. Analysis of the correlation between the distance measurements analysed in the present study, by sex and race

\begin{tabular}{|c|c|c|c|c|c|c|c|c|}
\hline & \multicolumn{2}{|c|}{ White males } & \multicolumn{2}{|c|}{ Black males } & \multicolumn{2}{|c|}{ White females } & \multicolumn{2}{|c|}{ Black females } \\
\hline & $r$ & $\mathbf{p}$ & $\mathbf{r}$ & $\mathbf{p}$ & $r$ & $\mathbf{p}$ & $r$ & $\mathbf{p}$ \\
\hline $\mathrm{AC}-\mathrm{BM} \times \mathrm{AC}^{-M L F_{s}}$ & 0.951 & 0.000 & 0.890 & 0.000 & 0.965 & 0.000 & 0.898 & 0.000 \\
\hline AC-BM $\times$ AC-MLF $_{m}$ & 0.876 & 0.004 & 0.843 & NS & - & - & 0.888 & NS \\
\hline $\mathrm{AC}-\mathrm{BM} \times \mathrm{AC}^{-M L F_{i}}$ & 0.948 & 0.000 & 0.954 & 0.003 & 0.971 & 0.001 & 0.967 & 0.000 \\
\hline$A C-B M \times A C-L L F_{R}$ & 0.903 & 0.000 & 0.402 & NS & 0.992 & NS & 0.532 & NS \\
\hline$A C-B M \times A C-L L F_{L}$ & 0.859 & 0.000 & 0.870 & 0.000 & 0.934 & NS & 0.670 & 0.048 \\
\hline${\mathrm{AC}-M L F_{\mathrm{s}}} \times \mathrm{AC}^{-M L F_{m}}$ & 0.943 & 0.001 & 0.815 & NS & - & - & 0.925 & NS \\
\hline AC-MLF $_{\mathrm{s}} \times \mathrm{AC}^{-M L F_{i}}$ & 0.934 & 0.000 & 0.874 & NS & 0.939 & 0.018 & 0.923 & 0.001 \\
\hline$A C-M L F_{s} \times A C-L L F_{R}$ & 0.935 & 0.000 & 0.485 & NS & 0.968 & NS & 0.375 & NS \\
\hline$A C-M L F_{S} \times A C-L L F_{L}$ & 0.831 & 0.000 & 0.806 & 0.002 & 0.999 & 0.023 & 0.807 & 0.015 \\
\hline$A C-M L F_{m} \times A C-L L F_{R}$ & - & - & - & - & - & - & - & - \\
\hline$A C-M L F_{m} \times A C-L L F_{L}$ & 0.925 & NS & - & - & - & - & 0.957 & NS \\
\hline$A C-M L_{F} \times A C-M L F_{m}$ & - & - & - & - & - & - & - & - \\
\hline$A C-M L F_{i} \times A C-L L F_{R}$ & 0.861 & 0.001 & 0.384 & NS & - & - & 0.627 & NS \\
\hline AC-MLF $F_{i} \times A C-L L F_{L}$ & 0.593 & NS & 0.940 & NS & 0.953 & NS & 0.831 & NS \\
\hline$A C-L_{L} F_{R} \times A C-L L F_{L}$ & 0.629 & 0.028 & 0.743 & 0.022 & 0.976 & NS & 0.936 & 0.006 \\
\hline
\end{tabular}

r Spearman's rho; NS — no statistical significance; AC — alveolar crest; BM — base of mandible; MLF — median lingual foramina (s - superior, $\mathrm{m}$ - middle, $\mathrm{i}$ - inferior); LLF — lateral lingual foramina (R - right, L- left); (-) distance not calculate

AC-MLF ${ }_{m}$ in the whole sample. The AC-MLF ${ }_{m}$ distance presented a perfect positive correlation with the AC- LLF $_{\mathrm{L}}$ distances in white males and black females.

The $A C-M F_{i}$ distance presented a strong or very strong positive correlation with the $A C-L_{R} F_{R}$ and AC-LLF distances for males and white individuals, while for females and black individuals the correlation between these distances presented no statistical significance. A strong or very strong positive correlation was observed between the $A C-L_{L} F_{R}$ and $A C-L L F_{L}$ distances. The AC-MLF $\mathrm{F}_{\mathrm{i}}$ distance presented a strong or very strong positive correlation with the $A C-L_{L} F_{R}$ for white males, and AC-LLF ${ }_{L}$ distances for black males, black females and white females.

The $A C-L_{L} F_{R}$ and $A C-L_{L} F_{L}$ distances presented a strong positive correlation for black females and white females.

\section{DISCUSSION}

Przystańska and Bruska [20] studied the content of foramina located in the area of the mandibular symphysis and observed the presence of a neurovascular bundle comprising one branch of the mylohyoideus nerve, a sublingual artery and accompanying veins; the artery was the largest diameter structure in this bundle. Due to the proximity of the blood vessels to the lingual cortical plate of the mandibular midline, there is a risk of surgical trauma in implant placement in this region since even a very small perforation of the mandibular cortical plate may cause bleeding [23]. The most likely reason for a haemorrhage is bleeding from a sublingual artery or its branches, secondary to lingual cortex perforation during osteotomy preparation for implant placement [11]. Woo et al. [24] report a case of haemorrhage resulting from an incision made too far lingual from the alveolar crest; the dissection was carried out supraperiosteally, resulting in laceration of the sublingual artery and hypoglossal nerve. According to Kalpidis and Konstantinidis [6], perforation of the lingual osseous cortical in the region of the canines during implant placement is quite a frequent complication.

Lingual foramina are constant structures in the anterior mandibular region. In our study, $99 \%$ of the mandibles presented at least $1 \mathrm{MLF}$, corroborating the studies of Rosano et al. [23] (100\%), McDonnel et al. [15] (99\%), Natsis et al. [19] (97\%), Liang et al. [12] (98\%); lower percentages were reported by Kim et al. [9] and by Przystańska and Bruska [2], with 58.8\% and $92 \%$, respectively. Liang et al. [12] observed that MLF tends to present singly in the mandible; in our study we observed that the MLF tends to present in pairs in the mandible, a similar finding to that reported by Rosano et al. [23]. We agree with Natsis et al. [19] 
and Liang et al. [12] when they report that the MLF tends to be located in the region superior to the GS in dentate individuals. In our study we observed that in completely edentate individuals the foramina are located in the regions superior and inferior to the GS. The high prevalence of MLF, and their location preferentially in the region superior to the GS, makes the area of the mandibular symphysis, superior to the GS, more vulnerable to complications during implant placement.

The AC-MLF distance is of greater clinical significance, since in completely edentate mandibles the $\mathrm{MLF}_{\mathrm{s}}$ is very close to the alveolar crest [19]. Natsis et al. [19] found an average value for this measurement of $18.28 \mathrm{~mm}$ in dentate individuals and $8.74 \mathrm{~mm}$ in edentate individuals in a Turkish population; Kawai et al. [8] reported that in Japanese individuals they found a mean value of $11.43 \mathrm{~mm}$ for this distance in a cone-beam computerised tomography study. In our study the mean value for the AC-MLF distance presented significant differences between dentate and edentate individuals, varying between $17.36 \mathrm{~mm}$ and $20.96 \mathrm{~mm}$ in dentate individuals and between $9.90 \mathrm{~mm}$ and $16.16 \mathrm{~mm}$ in edentate individuals. The values found in our study are similar to those reported by Natsis et al. [19] but quite different from those found by Kawai et al. [8], probably because these authors did not study dentate and edentate mandibles separately. In our study, dentate and edentate black males presented greater mean values for this distance, while dentate and edentate white females presented the lowest mean values. In males and white individuals, and more specifically white males, we found very significant differences between dentate and edentate individuals; the latter present greater proximity of the foramina to the alveolar crest, resulting in greater risk of neurovascular injury during surgical procedures in the anterior mandibular region. When we analysed the correlation between the AC-BM $\times$ AC-MLF (superior, middle and inferior) distances, we found that the distance from the $A C$ to the $B M$ is proportional to the distance from the $A C$ to the MLF (superior, middle and inferior), in other words, the greater the distance from the alveolar crest to the base of mandible, the greater will be the distance from the alveolar crest to the MLF, corroborating the finding reported by Natsis et al. [19]. We also found that the AC-MLF, AC-MLF $F_{m}$ and AC-MLF distances increase or diminish in proportion to one another. Thus mandibles with a shorter vertical dimension in the area of the symphysis present shorter AC-MLF distances; the same occurs in completely edentate mandibles, in which the MLF are located in a region closer to the alveolar crest than in dentate mandibles.

In our study the $B M-M L F_{s^{\prime}} B M-M L F_{m}$ and $B M-M L F_{i}$ measurements showed no significant differences between mandibles of dentate and completely edentate individuals. This contradicts the findings of Natsis et al. [19], who reported important differences in the BM-MLF distance, stating that this measurement was also affected by tooth loss. Liang et al. [12] found

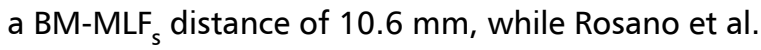
[23] found values of $12.5 \mathrm{~mm}$ for the BM-MLF $\mathrm{s}_{\mathrm{s}}$ dis-

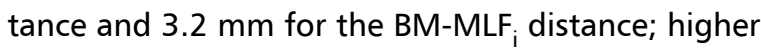
mean values were reported by Natsis et al. [19], of $18.28 \mathrm{~mm}$ and $16.21 \mathrm{~mm}$ for the BM-MLF ${ }_{\mathrm{s}}$ distance and $8.21 \mathrm{~mm}$ and $6.54 \mathrm{~mm}$ for the BM- MLF distance in dentate and edentate individuals, respectively. In our study the $\mathrm{MLF}_{\mathrm{s}}$ and the $\mathrm{MLF}_{\mathrm{i}}$ were located further inferior in the mandible than was reported by Natsis et al. [19] for the Turkish population, and further superior in the mandible than reported by Rosano et al. [23] and Liang et al. [12].

In our study we found at least $1 \mathrm{LLF}$ in either the left or right side in $82.5 \%$ of mandibles. In general LLF are located in the middle region both in dentate and completely edentate individuals; Natsis et al. [19], Przystańska and Bruska [20] and Liang et al. [12] found LLF generally located in the inferior third of the mandible. For Kim et al. [9], lateral foramina appeared more frequently on the right side $(32.9 \%)$ than the left side $(27.8 \%)$, corroborating the study by Liang et al. [12] who found $63 \%$ of LLF on the right side and $37 \%$ on the left side. Natsis et al. [19] state that LLF are usually bilateral; Przystańska and Bruska [20] report $36 \%$ and Liang et al. [12] report $48.4 \%$ of mandibles with bilateral presence of LLF. In our study LLF were found bilaterally in $49.2 \%$ of dentate mandibles, and were slightly more frequent on the left side, with no statistical significance. In completely edentate individuals LLF presented bilaterally in $50 \%$ of the mandibles and were slightly more frequent on the right side than on the left; however, there was no statistical difference between sides in any case. There is usually only one LLF in each hemimandible; however, up to 3 foramina per hemimandible were observed in our study. Przystańska and Bruska [20] report that they never found more than 2 foramina in each hemimandible, while Natsis et al. [19] say that they observed up to 4 foramina in each hemimandible. 
The mean value for the distance from the LLF to the GS varied between $10.63 \mathrm{~mm}$ and $13.77 \mathrm{~mm}$ for dentate mandibles and between $9.05 \mathrm{~mm}$ and $12.90 \mathrm{~mm}$ for edentate mandibles. Although completely edentate mandibles presented lower values for this distance, we did not observe a statistical difference between mandibles of dentate individuals and completely edentate individuals. Przystańska and Bruska [20] report higher mean values than found in our study, with a distance between the foramen and the midline of $15.4 \mathrm{~mm}$ (left) and $15.2 \mathrm{~mm}$ (right). In the present study, the AC-LLF $F_{R}$ distance varied between $24.39 \mathrm{~mm}$ and $24.92 \mathrm{~mm}$ for dentate mandibles and between $13.75 \mathrm{~mm}$ and $20.08 \mathrm{~mm}$ for edentate mandibles. In males, white individuals and white males the mean value found for edentate mandibles was around $11 \mathrm{~mm}$ lower than for dentate mandibles, from which we may conclude that in edentate individuals the LLF is closer to the alveolar crest than in dentate individuals, corroborating the findings of Natsis et al. [19]. When the correlation between the distances studied is analysed, we find that the AC-BM distance is proportional to the AC-LLF distance (right and left); i.e. a mandible with a smaller vertical dimension in the area of the symphysis (AC-BM distance) presents LLF less distant from the alveolar crest than a mandible with a larger vertical dimension in the area of the symphysis. In our study we also found symmetry in the distance from LLF, with the measurements of the $A C-L_{L} F_{R}$ and $A C-L L F_{L}$ distances increasing or diminishing in proportion.

Przystańska and Bruska [21] found neurovascular foramina over the inner surface of the alveolar region in $32 \%$ of the mandibles studied, with $98 \%$ located symmetrically; in 6 cases they found a single foramen on the midline. In our study we found LLF in the alveolar region in $67 \%$ of the sample, with bilateral LLF in $86.7 \%$ of cases. All the alveolar foramina were found in the lateral region, up to a maximum of 3 foramina in each hemimandible; however, the presence of a single foramen on the left and/or right was more frequent. Other authors report finding ALLF in $23.7 \%$ on the right side and $30.9 \%$ on the left side [19]; these percentages are lower than found in our study. Natsis et al. [19] found AMLF in $19.6 \%$ of mandibles and Murlimanju et al. [18] in 1.9\%; in our study we did not find any AMLF.

The morphology of edentate mandibles increases the risk of intraoperative complication in the anterior mandibular region [19]. After tooth loss, bone resorp- tion occurs in the lingual and buccal aspects [10]; as a result, in completely edentate individuals $\mathrm{MLF}_{\mathrm{s}}$ and LLF are significantly closer to the alveolar ridge than in dentate individuals. In our study we observed that the vertical dimension of the symphysis diminishes significantly in edentate individuals. No significant differences were found between sexes for the AC-BM distance, but there was a difference between races, with a greater mean value for this distance in white males than black males. We agree with Natsis et al. [19] when they say that the locations of LLF and MLF are affected by the dental state of the individual, since LLF and MLF almost reach the AC in mandibles of completely edentate individuals. In our study completely edentate white males presented $\mathrm{MLF}_{\mathrm{s}}$ up to $7 \mathrm{~mm}$ closer to the $A C$, and $M L F_{i}$ up to $10 \mathrm{~mm}$ closer to the $A C$, than dentate individuals.

When placing an implant, the surgeon must consider the anatomical conditions of the mandible in order to prevent early failure [25]. The amount and thickness of the cortical bone at the implant site must be analysed [17]. In edentate patients the thickness of the cortical bone is significantly less than in dentate mandibles [7]. Implants in mandibles with advanced resorption, when combined with poor, dense bone, must be considered potential risk situations for fixture placement. The length of the implant is related to failures in completely edentate patients; however, this condition is not observed in partially dentate patients, possibly due to better bone quality [3]. Many authors state that such a long implant is not necessary to reach the bicortical anchorage [5]; however, shorter implants $(7 \mathrm{~mm}$ ) present higher failure rates [3]. It is observed in the literature that implants of $15 \mathrm{~mm}$ to $18 \mathrm{~mm}$ have failed in completely edentate patients, with reports of important haemorrhagic accidents [14, 16, 22, 24]; in many case reports, the haemorrhage led to significant swelling and oedema of the floor of mouth and tongue, resulting in respiratory difficulty and obstruction of the airways due to elevation of the tongue against the palatal vault [24]. Careful assessment is recommended before using implants longer than $13 \mathrm{~mm}$ in the anterior mandibular region [23]. In our study we found a mean value for the AC-MLF distance of $9.90 \mathrm{~mm}$ and $10.54 \mathrm{~mm}$ for completely edentate mandibles of white males and white individuals, respectively. Proper surgical planning is therefore indispensable before implant placement, since shorter implants may be indicated in completely 
edentate individuals in order to avoid complications from injury to the neurovascular bundle.

\section{CONCLUSIONS}

The present study contributes detailed information about the location of lingual foramina by sex and skin colour; no other research was found in the literature providing detailed information on this subject. We conclude from our study that lingual foramina are constant structures in the anterior mandibular region. MLF are more frequent than LLF; they usually present in pairs when they are median and singly when they are lateral (in each hemimandible). Sexual and racial differences were not so marked in the present study; however, we observed that males and white individuals present lingual foramina in a region closer to the alveolar crest than females and black individuals. We also conclude that MLF generally present superior to the genial spine, with a significantly smaller distance from the alveolar crest to the $\mathrm{MLF}_{\mathrm{s}}$ in completely edentate than in dentate mandibles. In the present study, the mandibles with a shorter distance from the base of mandible to the alveolar crest presented MFL and LLF closer to the alveolar crest, implying a greater risk of complication during implant placement in the anterior mandibular region.

\section{Acknowledgements}

Department of Morphology and Genetics, Federal University of São Paulo, UNIFESP.

\section{REFERENCES}

1. Alves N, Cândido PL. Anatomia para o curso de odontologia geral e específica. 4ª Ed. Gen-Santos, São Paulo 2016.

2. Flanagan D. Important arterial supply of the mandible, control of an arterial hemorrhage, and report of a hemorrhagic incident. J Oral Implantol. 2003; 29(4): 165-173, doi: 10.1563/1548-1336(2003)029<0165:IASOTM> 2.3.CO;2, indexed in Pubmed: 12964796.

3. Friberg B, Jemt T, Lekholm U. Early failures in 4,641 consecutively placed Brånemark dental implants: a study from stage 1 surgery to the connection of completed prostheses. Int J Oral Maxillofac Implants. 1991; 6(2): 142-146, indexed in Pubmed: 1809668.

4. Hofschneider U, Tepper G, Gahleitner A, et al. Assessment of the blood supply to the mental region for reduction of bleeding complications during implant surgery in the interforaminal region. Int J Oral Maxillofac Implants. 1999; 14(3): 379-383, indexed in Pubmed: 10379111.

5. Ivanoff CJ, Gröndahl K, Bergström C, et al. Influence of bicortical or monocortical anchorage on maxillary implant stability: a 15-year retrospective study of Brånemark System implants. Int J Oral Maxillofac Implants. 2000; 15(1): 103-110, indexed in Pubmed: 10697944.
6. Kalpidis $C D$, Konstantinidis AB. Critical hemorrhage in the floor of the mouth during implant placement in the first mandibular premolar position: a case report. Implant Dent. 2005; 14(2): 117-124, indexed in Pubmed: 15968182.

7. Katranji A, Misch K, Wang HL. Cortical bone thickness in dentate and edentulous human cadavers. J Periodontol. 2007; 78(5): 874-878, doi: 10.1902/jop.2007.060342, indexed in Pubmed: 17470021.

8. Kawai T, Asaumi R, Sato I, et al. Classification of the lingual foramina and their bony canals in the median region of the mandible: cone beam computed tomography observations of dry Japanese mandibles. Oral Radiology. 2007; 23(2): 42-48, doi: 10.1007/s11282-007-0064-0.

9. Kim H, Choi B, Lee $H$, et al. Morphological study of the mental spine, lingual foramen and nutrient foramen and innominate foramen in Korean mandibles. Korean J Phys Anthropol. 1993; 6(1): 129-140, doi: 10.11637/ kjpa.1993.6.1.129.

10. Kingsmill VJ, Boyde A. Variation in the apparent density of human mandibular bone with age and dental status. J Anat. 1998; 192 ( Pt 2): 233-244, indexed in Pubmed: 9643424.

11. Kusum CK, Mody PV, Nooji D, et al. Interforaminal hemorrhage during anterior mandibular implant placement: An overview. Dent Res J (Isfahan). 2015; 12(4): 291-300, doi: 10.4103/1735-3327.161422, indexed in Pubmed: 26288617.

12. Liang $X$, Jacobs $R$, Lambrichts $I$, et al. Lingual foramina on the mandibular midline revisited: a macroanatomical study. Clin Anat. 2007; 20(3): 246-251, doi: 10.1002/ ca.20357, indexed in Pubmed: 16683247.

13. Lustig JP, London $\mathrm{D}$, Dor $\mathrm{BL}$, et al. Ultrasound identification and quantitative measurement of blood supply to the anterior part of the mandible. Oral Surg Oral Med Oral Pathol Oral Radiol Endod. 2003; 96(5): 625-629, doi: 10.1016/ S107921040300516X, indexed in Pubmed: 14600700.

14. Mason ME, Triplett RG, Alfonso WF. Life-threatening hemorrhage from placement of a dental implant. J Oral Maxillofac Surg. 1990; 48(2): 201-204, indexed in Pubmed: 2299461.

15. McDonnell $D$, Reza Nouri M, Todd ME. The mandibular lingual foramen: a consistent arterial foramen in the middle of the mandible. J Anat. 1994; 184 ( Pt 2): 363-369, indexed in Pubmed: 8014127.

16. Mordenfeld A, Andersson L, Bergström B. Hemorrhage in the floor of the mouth during implant placement in the edentulous mandible: a case report. Int J Oral Maxillofac Implants. 1997; 12(4): 558-561, indexed in Pubmed: 9274086.

17. Motoyoshi M, Inaba $M$, Ono $A$, et al. The effect of cortical bone thickness on the stability of orthodontic mini-implants and on the stress distribution in surrounding bone. Int J Oral Maxillofac Surg. 2009; 38(1): 13-18, doi: 10.1016/j.ijom.2008.09.006, indexed in Pubmed: 18963818.

18. Murlimanju BV, Prakash KG, Samiullah D, et al. Accessory neurovascular foramina on the lingual surface of mandible: incidence, topography, and clinical implications. Indian J Dent Res. 2012; 23(3): 433, doi: 10.4103/09709290.102252, indexed in Pubmed: 23059593. 
19. Natsis K, Repousi E, Asouhidou I, et al. Foramina of the anterior mandible in dentate and edentulous mandibles. Folia Morphol. 2016; 75(2): 204-210, doi: 10.5603/ FM.a2015.0090, indexed in Pubmed: 26431051.

20. Przystańska A, Bruska M. Anatomical classification of accessory foramina in human mandibles of adults, infants, and fetuses. Anat Sci Int. 2012; 87(3): 141-149, doi: 10.1007/s12565-012-0136-z, indexed in Pubmed: 22553081.

21. Przystańska A, Bruska M. Foramina on the internal aspect of the alveolar part of the mandible. Folia Morphol. 2005; 64(2): 89-91, indexed in Pubmed: 16121325.

22. Ratschew C, Czernicky W, Watzek G. Life threatening hemorrhage during placement of dental implants in the mandible. A case report. Deutsch Zahnärtztliche Zeitschrift. 1994; 49: 67-67.
23. Rosano G, Taschieri S, Gaudy JF, et al. Anatomic assessment of the anterior mandible and relative hemorrhage risk in implant dentistry: a cadaveric study. Clin Oral Implants Res. 2009; 20(8): 791-795, doi: 10.1111/j.16000501.2009.01713.x, indexed in Pubmed: 19489928.

24. Woo BM, Al-Bustani S, Ueeck BA. Floor of mouth haemorrhage and life-threatening airway obstruction during immediate implant placement in the anterior mandible. Int J Oral Maxillofac Surg. 2006; 35(10): 961-964, doi: 10.1016/j.ijom.2006.03.020, indexed in Pubmed: 16829038.

25. Worthington $\mathrm{P}$, Bolender $\mathrm{CL}$, Taylor TD. The Swedish system of osseointegrated implants: problems and complications encountered during a 4-year trial period. Int J Oral Maxillofac Implants. 1987; 2(2): 77-84, indexed in Pubmed: 3325415. 